MATEC Web of Conferences 32, 06001 (2015)

DOI: $10.1051 /$ matecconf $/ 20153206001$

(O) Owned by the authors, published by EDP Sciences, 2015

\title{
Linear encoder based low frequency inertial sensor
}

\author{
Christophe Collette ${ }^{1, a}$, Pierre Lambert ${ }^{1}$, Sylvain Hellegouarch ${ }^{1}$, Lionel Fueyo Roza ${ }^{1}$ and Kurt Artoos ${ }^{2}$ \\ ${ }^{1}$ BEAMS Department CP 165/56, Université Libre de Bruxelles, 50, av. F.D Roosevelt, 1050 Brussels, Belgium \\ ${ }^{2}$ Engineering department, CERN1211 Geneva 23, Switzerland
}

\begin{abstract}
For many applications, there is an increasing demand for low cost, high-resolution inertial sensors, which are capable of operating in harsh environments. Recently, a prototype of small optical inertial sensor has been built, using a Michelson interferometer. A resolution of $3 \mathrm{pm} / \sqrt{\mathrm{Hz}}$ has been obtained above $4 \mathrm{~Hz}$ using only low cost components. Compared to most state-of-the-art devices, this prototype did not contain any coil, which offers several important advantages, including a low thermal noise in the suspension and a full compatibility with magnetic environments (like particle collider). On the other hand, the Michelson is known to be tricky to tune, especially when one attempts to miniaturize the sensor. In this paper, we will propose a novel concept of inertial sensor, based on a linear encoder. Compared to the Michelson, the encoder is much more easy to mount, and the calibration more stable. The price to pay is a reduced resolution. In order to overcome this limitation, we amplify mechanically the relative motion between the support and the inertial mass. First results obtained with the new sensor will be discussed, and compared with the Michelson inertial sensor.
\end{abstract}

\section{Introduction}

Inertial sensors have been used for more than a century mainly to answer the needs of seismology, the science which studies the propagation of waves through the Earth. Depending on the frequency range of interest, three types of sensors are commonly used to measure seismic vibrations [1]: seismic accelerometers, geophones and broadband seismometers. A comparison of these inertial sensors can be found in [2]. For more than 30 years, seismometers have reached sufficient resolution and dynamic range to capture seismic signals at most location of the Earth surface in a broad frequency range extending typically from $1 \mathrm{~min}$ to $100 \mathrm{~Hz}$ (see e.g. [3-6]). However, there is still a continuous demand for high-end instruments, more efficient and better adapted to some specific applications. In this respect, recent developments in optical technologies offer interesting perspectives for novel inertial sensors. In the oil/gas and mining industry for instance, inertial sensors capable of operating in harsh environments (e.g. down-holes, boreholes) are needed, and optical seismometers without electronics and insensitive to temperature and high pressure [7-10] have been developed. In the field of security, miniature autonomous optical inertial sensors have been tested for the detection of detonation arising from nuclear tests conducted by countries engaged in nuclear proliferation [11-13].

Besides seismology and the aforementioned applications, there is also a demand for inertial sensors for precision

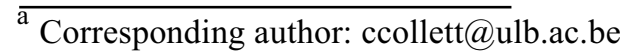

engineering and scientific experiments requiring a very stable environment [14]. Typical applications are: (i) tests and validation of space equipment on vibration-free space simulator (ii) isolation of lithography machines in the semiconductor industry (iii) reduction of vibrations of atomic force microscopes (support and sample) for increasing their resolution (iv) stabilization and isolation of large instruments dedicated to extreme experimental physics, like gravitational wave interferometric detectors or future particle colliders. In these systems the immunity to environmental disturbances is obtained by actively cancelling the structural vibration measured by inertial sensors [15]. A few other prototypes of optical inertial sensors and seismometers have been developed and reported in the literature. They are based on Fabry-Perot interferometer [22], fiber interferometer [19-21], triangulation system [23], fiber Bragg grating [24,25], optical encoder [26] or grating sensor [27]. More recently, optical accelerometers have been proposed for measuring the mechanical vibration of gravitational wave detectors [16-18]. However, to the best of the authors' knowledge, there is no commercial seismometer capable of fulfilling the requirements for applications in advanced active vibration isolation systems, i.e. small, compatible with a magnetic environment, with a sub-nanometer resolution and dynamic range as tentatively represented in the Fig. 1 by the area "new sensor".

The paper is organized as follows: The Non-magnetic Optical inertial Sensor (NOSE) is presented in Section 2. Section 3 presents the performance of a linear encoder,

This is an Open Access article distributed under the terms of the Creative Commons Attribution License 4.0, which permits unrestricted use, distribution, and reproduction in any medium, provided the original work is properly cited. 
selected as a good candidate for being integrated in an inertial sensor. Section 4 presents two prototypes of mechanical amplifiers, and section 5 draws the conclusions and directions for improvements.
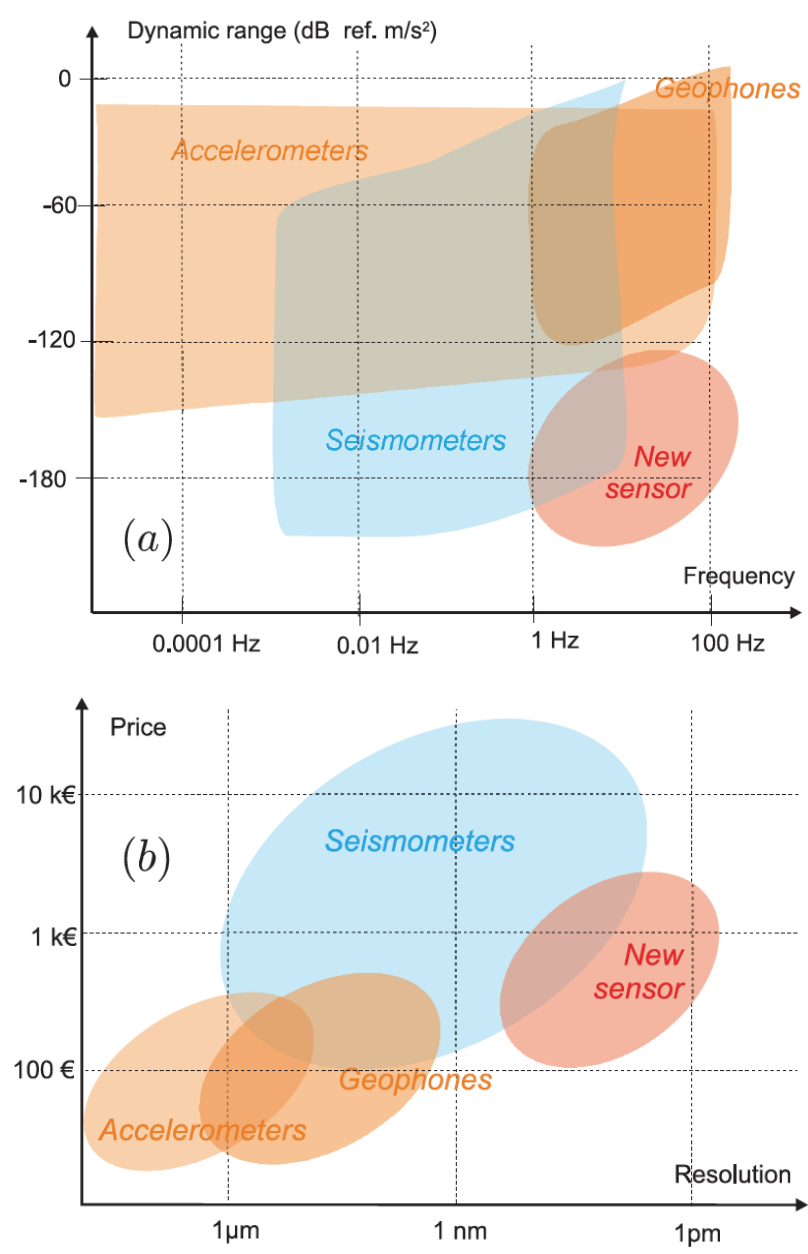

Figure 1 Comparison of inertial sensors. (a) Dynamic range vs. frequency; (b) Price vs. resolution.

\section{NOSE}

A picture of NOSE prototype is shown in Fig. 2. The mechanical part consists of a horizontal pendulum, connected to a rigid frame through a flexural joint, made of $\mathrm{CuBe}$ alloy. A leaf spring, made of the same alloy, is used to adjust the equilibrium position of the inertial mass and compensate for gravity. The oscillator is characterized by an inertial mass $m=0.055 \mathrm{~kg}$, a principal resonance frequency $f_{0}=6 \mathrm{~Hz}$ (tunable) and spurious resonances above $100 \mathrm{~Hz}$. NOSE does not contain any loaded coil, which was found to offer several advantages, including compatibly with magnetic environments and a low thermal noise in the suspension (Brownian motion) [28].

In order to measure the relative displacement between the inertial mass and the support, we have developed a sensor based on a Michelson interferometer, adapted to enable the measurement of both quadratures of the signals as in $[7,29]$.

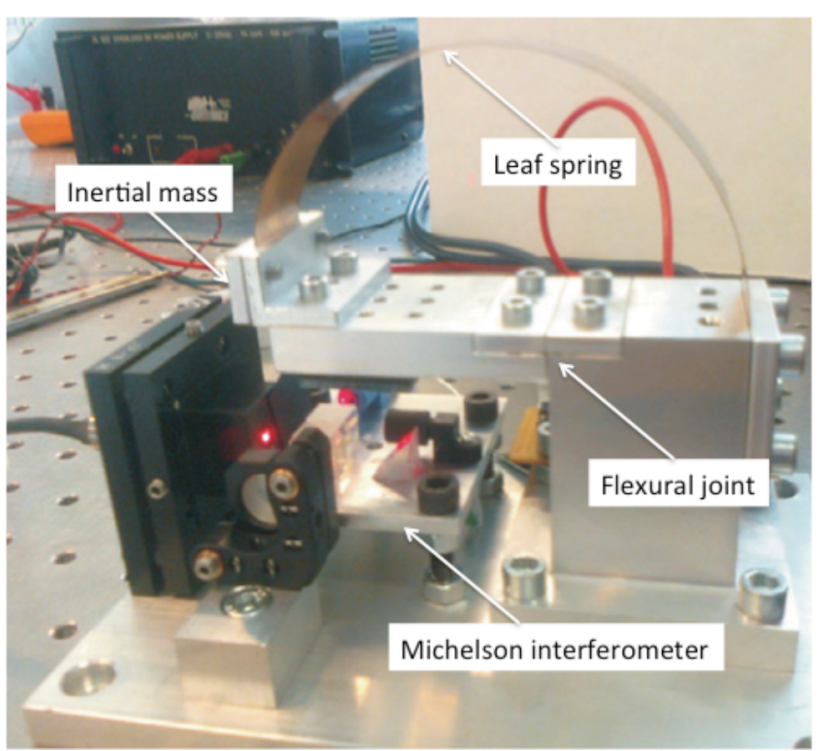

Figure 2 Picture of the interferometric inertial sensor NOSE.

Figure 3 shows the experimental amplitude spectrum of the interferometer noise (blue dashed-dotted line), expressed in physical units of $[\mathrm{m} / \sqrt{ } \mathrm{Hz}]$. It has been obtained in two steps. In the first step, we manually slightly moved one mirror in order to obtain the parameters of the Lissajous figure for the calibration. In a second step, we have recorded the signal without disturbing the interferometer, and used the parameters from the first step to express the photodiode signals as sensor noise in displacement units. It results in a resolution of $3 \mathrm{pm} / \sqrt{\mathrm{Hz}}$ above $1 \mathrm{~Hz}$, and $20 \mathrm{pm} / \sqrt{\mathrm{Hz}}$ above $0.3 \mathrm{~Hz}$ [30].

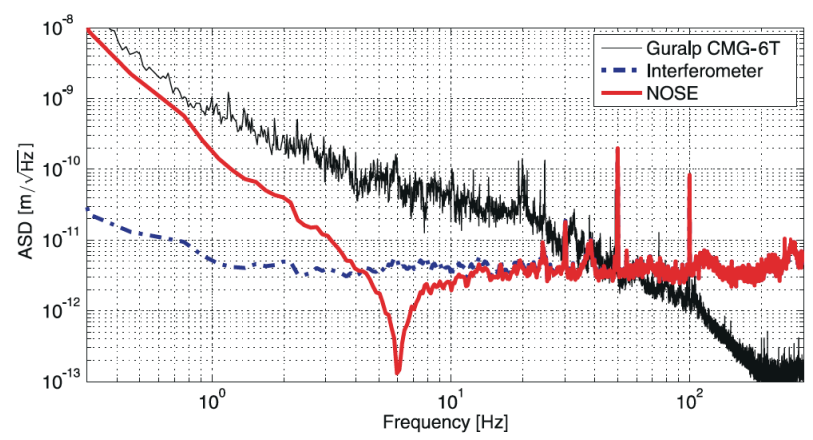

Figure 3 Comparison of sensor experimental resolution: Guralp CMG-6T (black line, obtained from two sensors side by side placed in a quiet environment); resolution of our NOSE prototype, measured by blocking the inertial mass; estimated resolution of NOSE (red line) [30].

Including the inertial mass dynamics, the resolution remains $3 \mathrm{pm} / \sqrt{\mathrm{Hz}}$ above $4 \mathrm{~Hz}$ (red line in Fig.3). Besides this performance, a possible weakness of NOSE may be that the calibration of the interferometer fluctuate over time, due to misalignment of the optical components. In the next sections, we will investigate the possibility to replace the Michelson interferometer by an linear encoder. 


\section{Linear encoder test bench}

The linear encoder considered is a LIP281 from Heidenhain. In order to check the calibration, it has been mounted on a test bench shown in Fig. 4, along with a Keyence LC-2440 and a geophone GS-11D. Both the LIP 281 and the LC-2440 measure the motion of the inertial mass of the mechanism. The mechanism has no spurious resonance below at least $500 \mathrm{~Hz}$. The LIP 281 signals have been amplified (INA118) to avoid ADC noise of the 16 bits recorder dSpace DS1103. Signals have been recorded with a sampling frequency of $10 \mathrm{kHz}$.

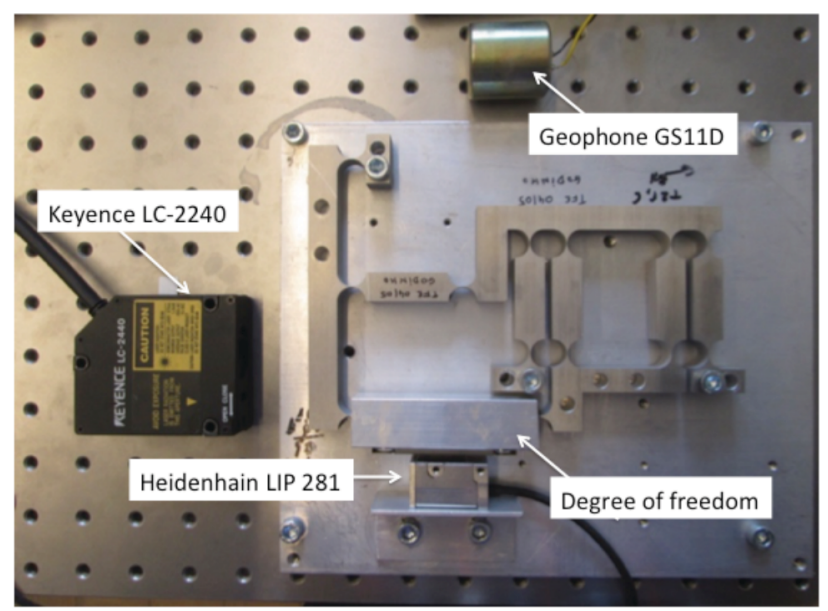

Figure 4 Picture of the experimental set-up

Figure 5 shows the Amplitude Spectral Densities (ASD) of the signals measured by the three sensors. In the frequency range around the resonance of the mechanism (around $7 \mathrm{~Hz}$ ) the ASD of the LC-2440 and of the LIP 281 are superimposed. Above $10 \mathrm{~Hz}$, the LIP 281 and the GS11D sense the same motion while the LC-2440 is dominated by ADC noise.

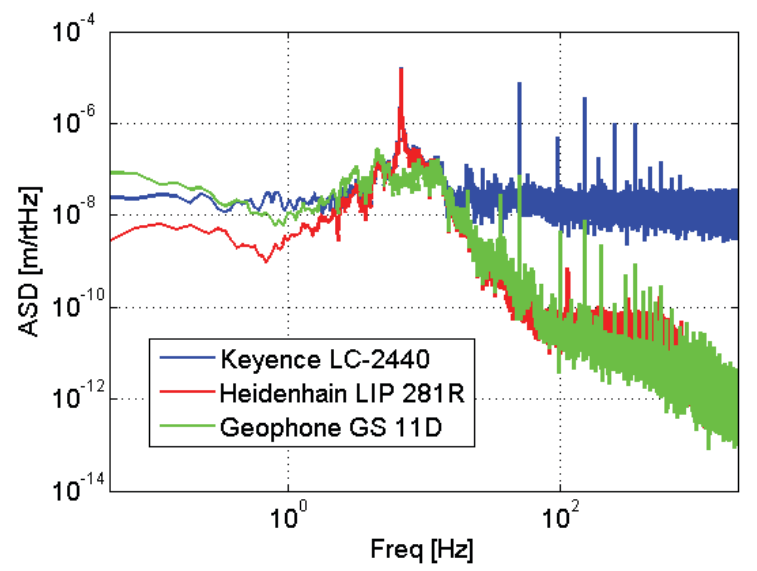

Figure 5 Signal calibration: LIP and LC are identical above the noise. Geophone is also identical between $10 \mathrm{~Hz}$ and $20 \mathrm{~Hz}$.

The resolution curves shown in Fig. 6 have been obtained when the mechanism is blocked. Two amplifications are visible. The first one is between $5 \mathrm{~Hz}$ and $100 \mathrm{~Hz}$; the second one is between $300 \mathrm{~Hz}$ and $600 \mathrm{~Hz}$. It is presumably inherent to the sensor itself. The second can be due to resonances in the mount of the sensor head. It can be noticed that the value at high frequency is below $10^{-12} \mathrm{~m} / \sqrt{ } \mathrm{Hz}$,

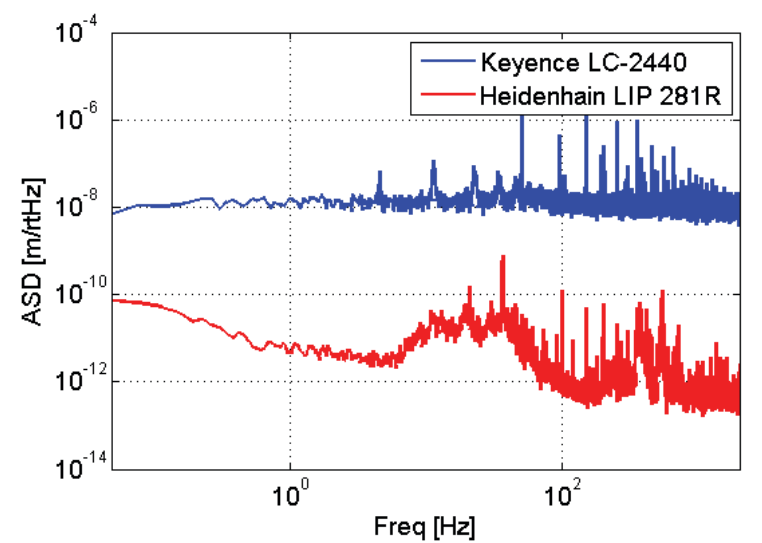

Figure 6 Resolution, i.e. signal when the inertial mass is blocked

The integrated RMS value shows a resolution of $0.3 \mathrm{~nm}$ below $4 \mathrm{~Hz}$.

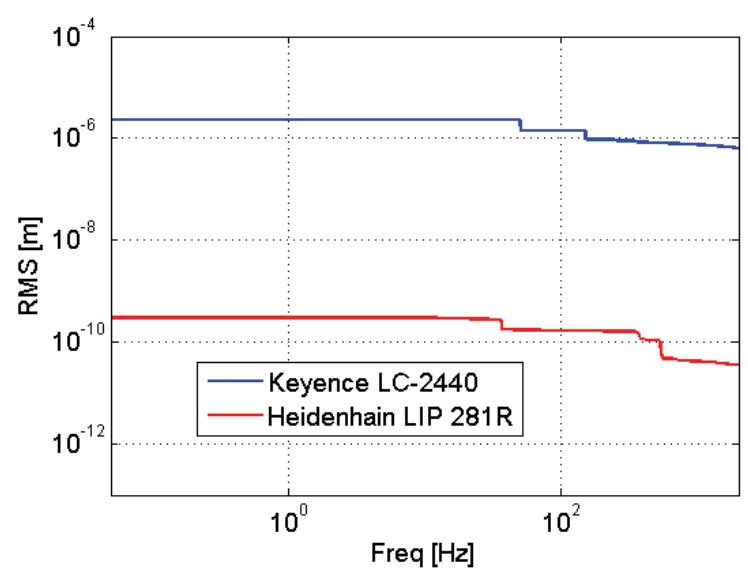

Figure 7 Integrated RMS value of the resolution

\section{Inertial mechanical amplifier}

As we have seen, the linear encoder is very promising compared to the Michelson interferometer. However, a slight amplification of the relative motion between both encoder parts and the ground motion would be useful. We therefore propose two mechanical amplification structures described in the following.

\subsection{First concept}

The first concept shown in Fig. 8 consists in a rod balanced by two masses. M plays the role of the inertial mass and $\mathrm{m}$ represents the mass of the linear scale. The rod is connected to the ground trough an elastic hinge developing a restoring torque $T=-\mathrm{K} \alpha$. Due to the ground vibration $u$, the rod is tilted with an angle $\alpha$. Considering the dynamic equation of the rod, we can write $\ddot{\alpha}$ as a function of $\ddot{u}$ and write the amplification factor of the mechanism as $\alpha l_{2} / u$. Indeed, it can be assumed that, above the resonance frequency of the 
pendulum, the fixed point of the mechanism is located close to $M$, and the relative motion $x_{m}-u$ measured by the encoder will correspond to the relative motion $\mathrm{x}-\mathrm{u}$ amplified by the suspended bar.

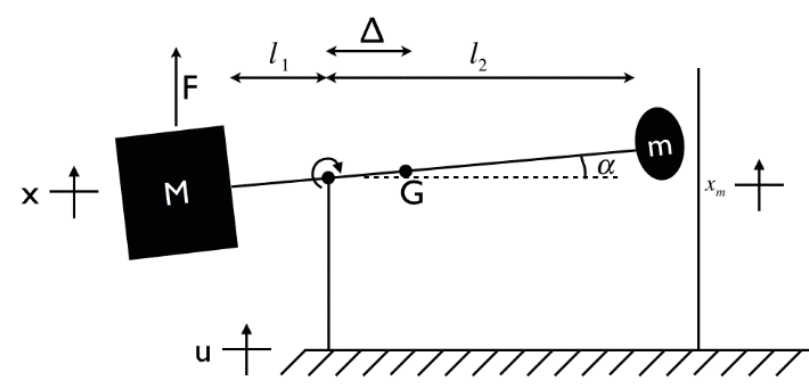

Figure 8 First concept of inertial mechanical amplifier.

After some manipulations, it can be shown that, above the resonance frequency of the oscillator, the amplification factor is:

$$
H_{1}=\frac{M l_{1} l_{2}-m l_{2}^{2}}{M l_{1}^{2}+m l_{2}^{2}}
$$

or, upon defining $\mu=M / m$ and $x=l_{I} / l_{2}$,

$$
H_{1}(\mu, x)=\frac{\mu x-1}{\mu x^{2}+1}
$$

The amplification is shown in Fig. 9 as a function of the mass ratio $\mu$ and of the length ratio $x$.

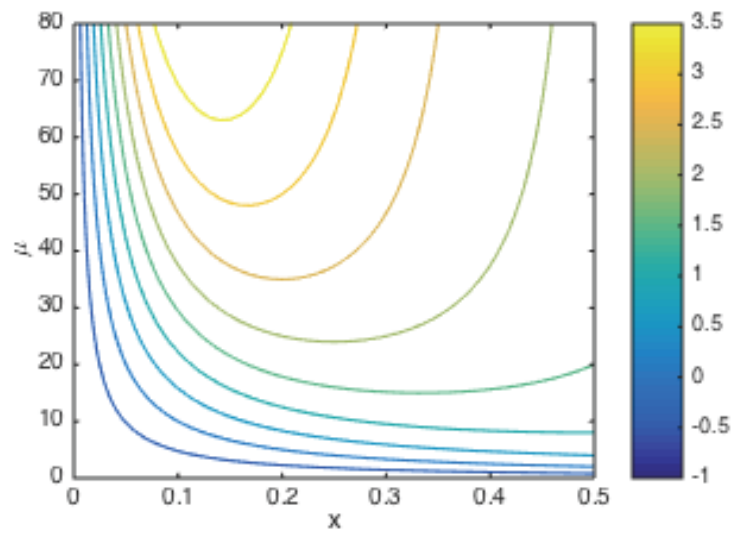

Figure 9 Mechanical amplification obtained using the first concept as a function of the mass ratio $\mathrm{M} / \mathrm{m}$ and of the length ratio $l_{2} / 1_{1}$.

A first weakness of the design is that, as $m$ has a circular trajectory, the linear scale will be misaligned for large amplitudes. If we assume that the maximum amplitude of vibration of the ground is 10 microns, so the maximum amplitude to be measured is 20 microns, with $l_{2}=0.16 \mathrm{~m}$, the angular misalignment will be of $0.125 \mathrm{mrad}$. We will assume that this angular misalignment is tolerable for the measuring device. A second weakness is that it is difficult to combine a large amplification with a good rejection of spurious resonance modes, due to the lack of stiffness in the design.

\subsection{Second concept}

Figure 10 shows the working principle of the second amplification mechanism. It consists of a pendulum, on which is attached a mass $M$. Due to ground vibrations $u$, this pendulum tilts by an angle $\Theta$. A second pendulum is cinematically coupled to the first one, through a rigid vertical link. The coupling ensures that $\Theta l_{3}=\theta l_{1}$. Again, $m$ represents the mass of the linear scale, used to measure the relative displacement $y=\theta l_{2}$.

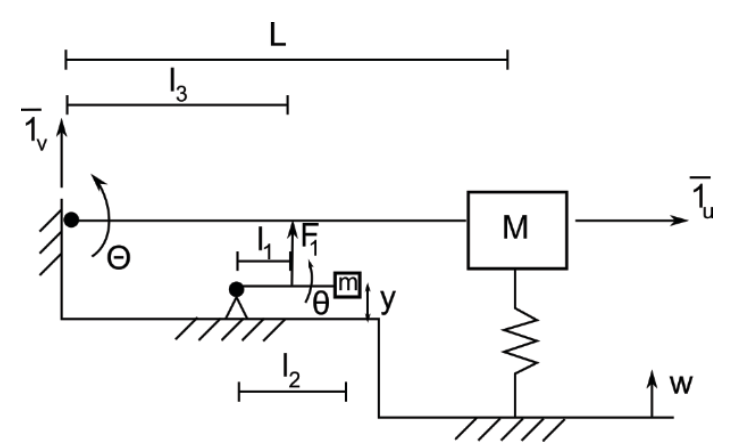

Figure 10 Second concept of inertial mechanical amplifier.

After some basic manipulation, the amplification factor $\theta l_{1} / u$ above the resonance frequency of the oscillator is found to be

$$
H_{2}=\frac{M L l_{1} l_{2} l_{3}+m l_{2}^{2} l_{3}^{2}}{M L^{2} l_{1}^{2}+m l_{2}^{2} l_{3}^{2}}
$$

Defining $\mu=M / m, D=L / l_{3}$ and $d=l_{2} / l_{1}$ and $x=D / d, H_{2}$ can be rewritten

$$
H_{2}(\mu, x)=\frac{\mu x+1}{\mu x^{2}+1}
$$

Note well that due to the topology difference in both mechanism, $l_{1}$ and $l_{2}$ does not represent the same quantity in both mechanisms. Hence, $x$ is also different. Again, the amplification is shown in Fig. 11 as a function of the mass ratio $\mu$ and of the length ratio $x$.

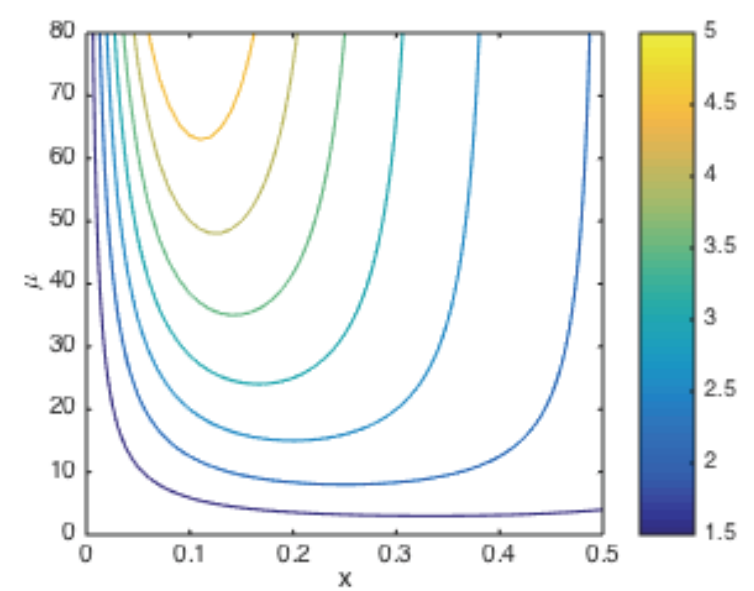

Figure 11 Mechanical amplification as a function of the mass ratio $\boldsymbol{\mu}$ and the length ratio $\mathrm{x}$. 
Figure 12 shows a CAD view of the prototype that has been developed on the basis of this second concept. A parallelogram has been used to reduce the inclination of the bar on which will be mounted the linear scale. After optimization, the prototype will be manufactured and tested.

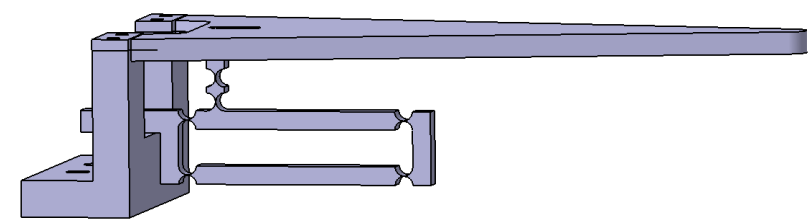

Figure $12 \mathrm{CAD}$ view of the prototype of mechanically amplified inertial sensor.

\section{Conclusions}

This paper has presented the status of an ongoing effort toward the development of a small optical inertial sensor, with a sub-nanometer resolution and robust to magnetic field and radiation. In the first part of the paper, the performance of the coil-free optical sensor NOSE has been presented. In order to balance the potential lack of robustness of the interferometer, a linear encoder has been considered as an alternative sensor to measure the relative motion between the support and the inertial mass. Compared to the interferometer, the encoder is much less sensitive to mounting error, but its resolution has to be increased by at least a factor 2. Two concepts of mechanical amplifier have been proposed and compared. A prototype based on the second concept has been designed. After optimization, it will be soon manufactured and tested.

\section{Acknowledgments}

The authors gratefully acknowledge the Brussels Capital Region and the FNRS for funding and supporting this research.

\section{References}

1. E. Wienlandt, Seismometry, in: W.H.K. Lee, H. Kanamori, P.C. Jennings, C.Kisslinger (Eds.), International Handbook of Earthquake and Engineering Seismology, Part A, Academic Press, Amsterdam, pp. 283-304 (2002).

2. C. Collette, S. Janssens, P. Fernandez-Carmona, K. Artoos, M. Guinchard, C.Hauviller, A. Preumont, Bull. Seismol. Soc. Am. 102 (4) 1289-1300 (2012).

3. H.E. Sheffield, IEEE Trans. Inst. Meas. 13 2-7 (1964).

4. C. Teupser, A. Plesinger, Phys. Earth Planet. Inter. 18 58-63 (1979).

5. M.J. Usher, R.F. Burch, C. Guralp, Phys. Earth Planet. Inter. 18 38-50 (1979).

6. E. Wielandt, G. Sterkeisen, Bull. Seismol. Soc. Am. 72 (1982)
7. M. Zumberge, J. Berger, J. Otero, E. Wielandt, Bull. Seismol. Soc. Am. 100 (2) 598-605 (2010).

8. J. Otero, Development and Characterization of an Observatory-class, Broad-band, Non-Fedback, LeafSpring Interferometric Seismometer (PhD thesis), University of California, San Dego (2009).

9. M. Zumberge, J. Berger, E. Wielandt, Optical seismometer, US patent 2012/0247213 A1 (2012).

10. USSI. http://www.ussensorsystems.com/USSIGeophone-brochure.pdf

11. D. Carr, G. Bogart, S. Goodman, P. Baldwin, D. Robinson, A laser interferometric miniature seismometer, Monitoring Research Review: GroundBased Nuclear Explosion Monitoring Technologies (2008).

12. D. Carr, P. Baldwin, S. Knapp-Kleinsorge, H. Milburn, D. Robinson, pp. 268 (2010).

13. D. Carr, P. Baldwin, S. Knapp-Kleinsorge, H. Milburn, D. Robinson, Monitoring Research Review: Ground-Based Nuclear Explosion Monitoring Technologies (2011).

14. P. Shore, C. Cunningham, D. DeBra, C. Evans, J. Hough, R. Gilmozzi, H. Kun-zmann, P. Morantz, X. Tonnellier, CIRP Ann. Manuf. Technol. 59 694-716 (2010).

15. C. Collette, S. Janssens, K. Artoos, Recent Patents Mech. Eng. 4 212-219 (2011).

16. F. Acernese, G. Giordano, R. Romano, R. De Rosa, F. Barone, Rev. Sci. Instrum. 79074501 (2008).

17. F. Acernese, G. Giordano, R. Romano, R. De Rosa, F. Barone, Nucl. Instrum. Methods Phys. Res. A 617 457-458 (2010).

18. F. Acernese, G. Giordano, R. Romano, F. Barone, in: 15th World Conference in Earthquake Engineering, Lisbon, Portugal, (2012).

19. D. Gardner, T. Hofler, S. Baker, R. Yarber, S. Garrett, J. Lightwave Technol. 5 (7) 953-960 (1987).

20. A. Araya, K. Kawabe, T. Sato, M. Mio, K. Tsubono, Rev. Sci. Instrum. 64 (5) 1337-1341 (1993).

21. A. Araya, K. Sekiya, Y. Shindo, in: Symposium on Underwater Technology and Workshop on Scientific Use of Submarine Cables and Related Technologies, pp. 245-248 (2007).

22. I. Littler, J. Chow, D. Mcclelland, WO2010057247 (2010).

23. D. Chatrefou, EP354882 (2003).

24. B. Levine, US7714271, (2007).

25. B. Levine, WO201150275 (2011).

26. R. Cahill, E. Udd, US4471659 (1984).

27. B. Merchant, M. Okandan, An Interferometric, pp. 352 (2009).

28. P.R. Saulson, Phys. Rev. D 42 (8) 2437-2445 (1990).

29. M.J. Downs, K.W., Appl. Mech. Mater. 365 85-88 (1979).

30. C. Collette, F. Nassif, J. Amar, C. Depouhon, S.-P. Gorza, Sensors and Actuators A: Physical, 224, 7277 (2015). 